\section{CAN-4, an advanced author language for CAI, computer-based testing and psychological experimentation: PDP-9 implementation}

\author{
STACY CHURCHILL, LARS NAESS, and WILLIAM P. OLIVIER \\ The Ontario Institute for Studies in Education, Toronto, Ontario, Canada
}

The effective use of computers for tutorial (programmed instruction) teaching and on-line testing requires that curriculum authors have powerful special-purpose languages. One such language, CAN-4, has been designed and implemented to run on the PDP-9. The language includes advanced scoring, data recording, computation, timing, and file control facilities. The language and its implementation are described in relation to the instructional problems which the facilities are designed to meet.

\section{INTRODUCTION}

The effective use of computers for tutorial (programmed instruction) teaching requires that curriculum authors have powerful special-purpose languages. Quite similar language facilities are required for interactive computer-based testing and for some $t y$ pes of computer-controlled psychological experimentation.

The Department of Computer Applications of The Ontario Institute for Studies in Education (OISE) has carried out since 1967 a program of research and development in the field of computer aids to instruction. An important result of the program has been the design and implementation of a series of course authoring languages to which we have given the acronym CAN, for "Completely Arbitrary Name." The latest language in the series, CAN-4, provides a powerful facility for the major types of uses mentioned above: computer-assisted instruction, computer-based testing, and psychological experimentation.

\section{IMPLEMENTATION DESCRIPTION}

The CAN-4 implementation consists of (1) a preprocessor for source files (PROCS), (2) an interpreter program which executes source files and maintains students records (CAN or CANP), (3) a program (PROCTR) which creates a student registration file, and (4) a program (SEPS) to select, copy, and display items from the student performance file. The interrelationship of these programs is shown in Figs. 1 and 2. Typically, the

The authors acknowledge the important contribution of D. Ensor and D. Stansfield, who designed the early CAN-1 language from which the basic spirit of CAN-4 is borrowed. In response to requirements specified by W. P. Olivier, Ensor drafted the first specification of CAN-4, which has since been extensively expanded and revised by W. P. Olivier and S. Churchill. L. Naess, assisted by V. Grant, was chiefly responsible for the PDP-9 coding of the interpreter. data input and file maintenance routines are run at irregular intervals, whereas the interpreter is theoretically available at all times for use by students. All programs run in an $8 \mathrm{~K}$ user area under either the keyboard monitor or the TSS/9 timesharing monitor written at The OISE (Ensor, 1969; McLean, 1970).

\section{Program Debugging}

Figure 1 illustrates the sequence of operations by which a $\mathrm{CAN}-4$ source program is entered into the system, either through the card reader of a UNIVAC 1004 interfaced to the PDP-9 or through the Teletype, scanned for errors, and finally debugged by the author from a student console.

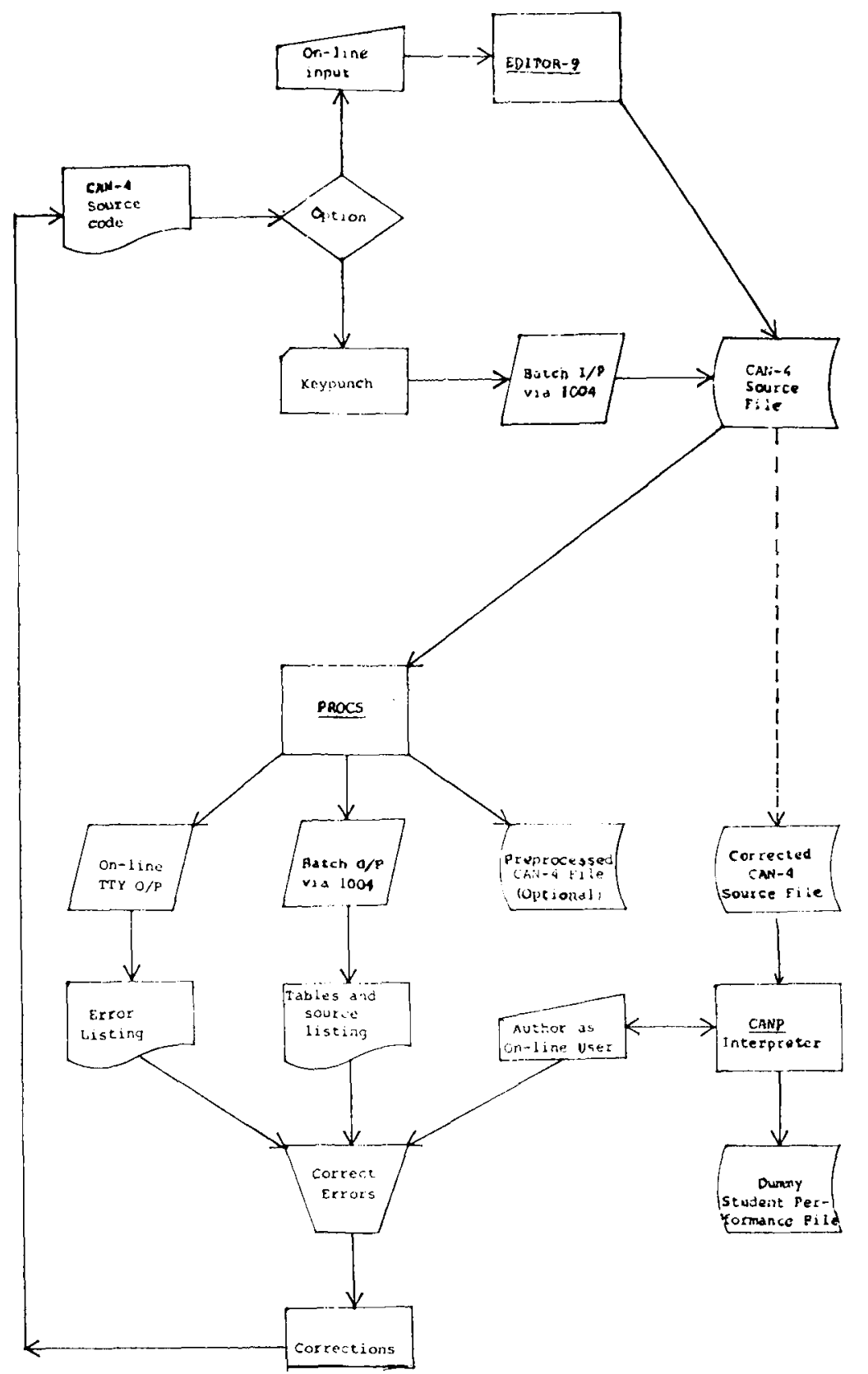

Fig. 1. Program entry and debugging procedures for CAN-4. 


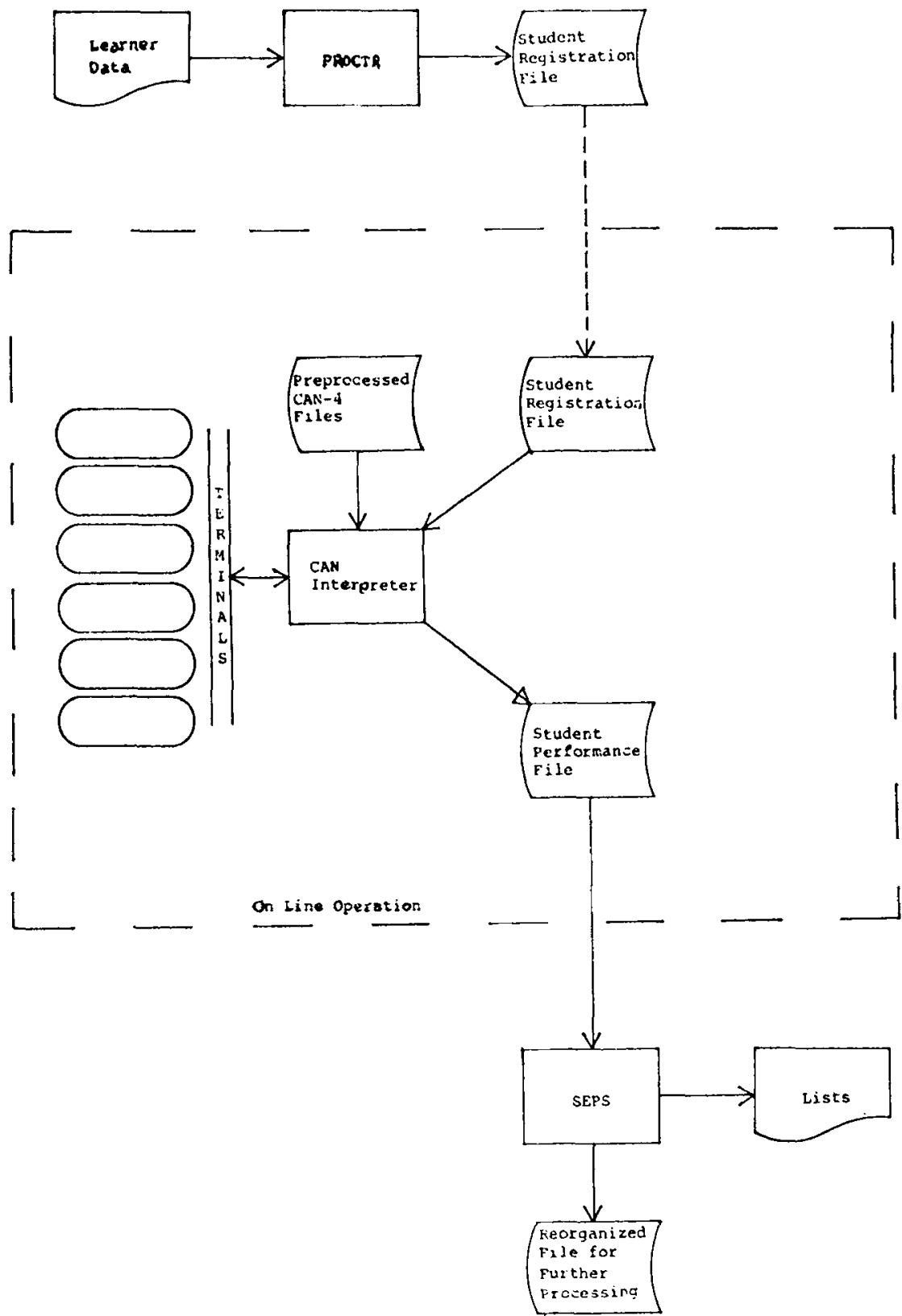

Fig. 2. File maintenance procedures and run-time operation of CAN interpreter for CAN-4.

Program debugging proceeds in two phases. First, using the system editor, the author corrects syntactic errors flagged by the preprocessor and, if no errors are found, the program is ready for logical debugging. For this purpose, a slightly modified version (CANP) of the CAN-4 interpreter is available. The version uses the unpreprocessed source file. It does not maintain the student registration file and creates student performance records under a dummy student name. (The name is that of the source program being used.)

\section{Preprocessor and Interpreter}

The preprocessor operates in two modes. In the debugging mode it scans runs under the TSS/9 timesharing monitor. At present this accommodates six simultaneous users (console Teletype and five terminals through an LT19). Each individual user interacts with a separate "copy" of the interpreter, which is swapped in and out by the timesharing system. Response delays are generally less than 1 sec, except when a new program file is accessed (about $3 \mathrm{sec}$ ). Each copy of the interpreter may access the following files: (1) any of the preprocessed $\mathrm{CAN}-4$ procedure files stored on the RBO9 disk, (2) a student registration file, and (3) student performance file. Branching may occur between different procedure files. In addition, the procedure file may specify the use of a fourth type of file, a data file, as input to the procedure; for example, "content" materials may be used as input and presented according to a predefined stratagem coded in CAN-4.

\section{File Maintenance}

Fig u re 2 show s the interrelationships between the main CAN-4 interpreter and the file maintenance programs, PROCTR and SEPS. Two principal files are maintained by the system: the student registration file and the student performance file. The registration file is created by the program PROCTR. Each record contains a student's name together with system initialization information. The storage of initialization information serves a double purpose: First, the author may cause the CAN-4 interpreter at any time during execution to store the exact status of the system; in case a student interrupts a lesson without completing it, this information can be used for a subsequent restart. Secondly, if so desired, a pseudoset of restart status information may be stored before a student signs on so that he will, upon initial entry, be directed automatically to a suitable starting point; in other words, it provides the ability to "assign" work to individuals.

The student performance file is a errors which are flagged by an appropriate printout. As an optional output, an analytical table of statement labels and variables and a complete source program listing may be prepared for printing in batch mode. In the second mode, which is for operational use of fully debugged CAN-4 programs, PROCS prodires all the outputs mentioned above and, in addition, a preprocessed CAN-4 file. The preprocessed file consists of the original source file to which has been added an analytical table of statement labels and associated disk addresses, used by the interpreter to speed its operations.

As mentioned above, the interpreter raw log of items recorded according to author commands in the CAN-4 source program. Using the $\mathrm{RD}$ (record data) command, the author may select any appropriate information to record on the student's performance. As an aid to program revision, an instruction (RU) may be used which causes all subsequent unanticipated student responses in a program to be recorded automatically for later analysis.

\section{INTRODUCTION TO THE} LANGUAGE STRUCTURE

The best way to introduce the CAN-4 language is to begin with an 
Table 1

Sample Frame of Programmed Instruction Coded Using Only a Limited Subset of Four CAN-4 Operation Codes: T, A, U, G

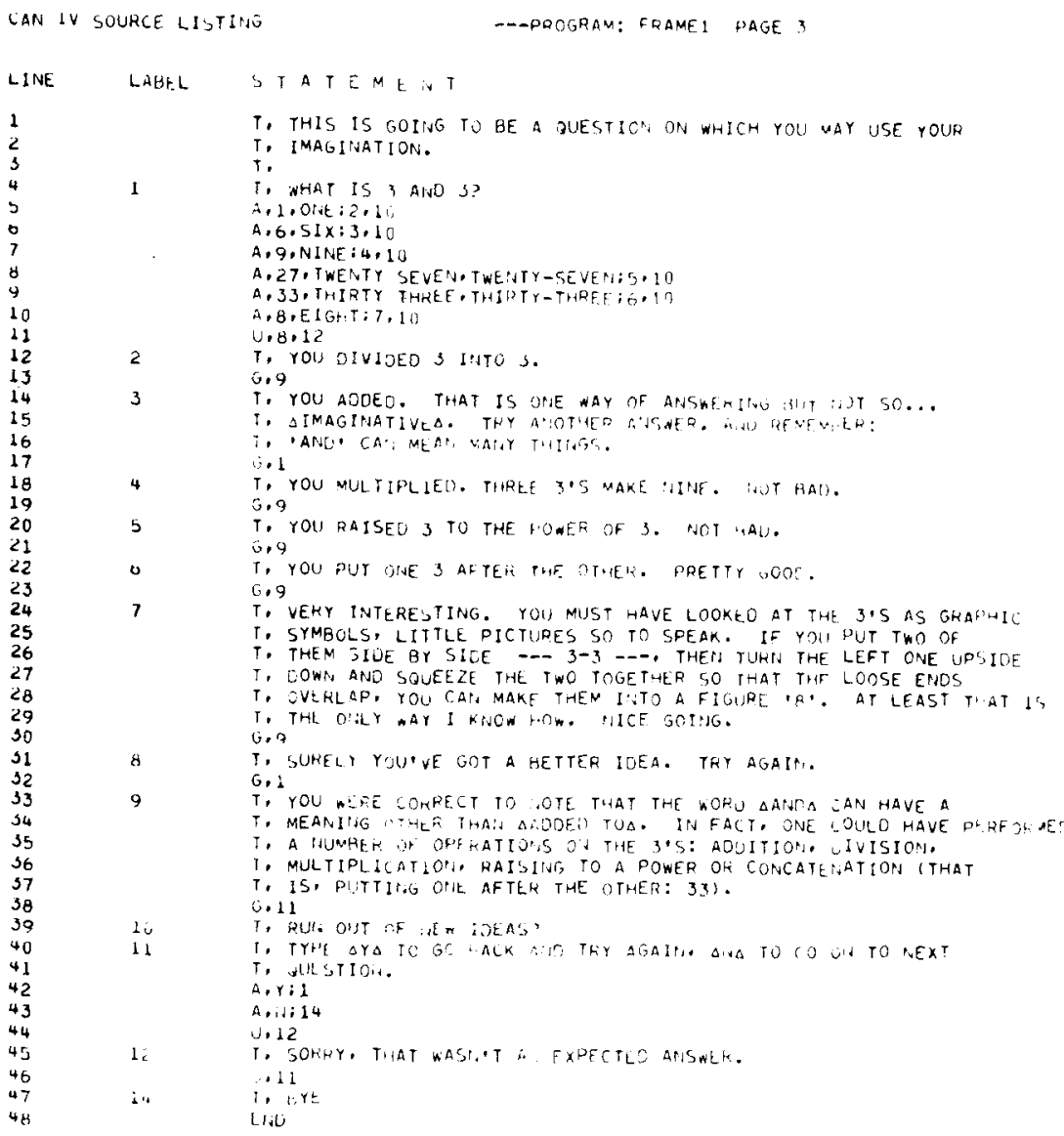

educational problem: How can one structure a programmed instruction sequence so that it may be administered by computer? A simple example of such a sequence, a programmed instruction "frame" coded in CAN-4 (Table 1) provides a basis for understanding the structure of the language.

The frame consists of the following parts: a test for presentation to the student, ending with a question; a so-called "answer list," answers which the author anticipates may be given by the learner; and, associated with each anticipated answer, an appropriate reply. The example shows how these might be coded, using a minimal subset of CAN-4: the operation codes $\mathrm{T}$ (text for presentation), A (anticipated answer), U (unanticipated answer), and $\mathbf{G}$ (go to). Each line has an operation code, which may be preceded by a numerical branch address or a text. The language is free format with commas and semicolons as delimiters in the field following the operation code. Lines are terminated by a carriage return.

In a typical interaction, the program would type on a Teletype the three lines of text ending with the question: "What is 3 and 3?" Upon encountering the A operation code, the program would switch to a state of waiting for the student response. If the student responded with the digit " 6 " or the letters "SIX," the response would be compared against each successive answer in the list until a match occured; in this case, it would be the second $A$ instruction, and a branch would occur to Statement 3 where a message would be printed out asking the student to try another answer. The $G$ (go to) instruction would cause a branch branch back to the beginning of the answer list, where a new response would be awaited.

The question might be asked: What if the student replies " 6 " for a second time? In fact, a match would occur on the second $A$ instruction-as before-but, this time, the second branch address after the semicolon would be used. This is also the way in which the second branch addresses in the other answer-list instructions function. On the other hand, if the learner had responded with, say, the digit " 5 ," no match would have occurred on any of the A instructions and the $U$ operation code would have been encountered, causing a branch to Statement 8.

The example illustrates four major language characteristics. The first is automatic acceptance of a student response: Upon encountering an answer processing operation code-the $A$, in this case-after an output, the interpreter causes the system to go into an input state to await a student response. The second feature is an automatic scan and match: The student response is automatically compared to the list of anticipated answers in each answer-processing instruction, until a match is found or until the list ends with one of the instructions-the $U$, in this case-intended to handle all unanticipated responses. Thirdly, an automatic branch takes place when a match is found or the end of the list is reached; the branch ordinarily occurs to the first address given following the semicolon. A fourth feature is the keeping of an automatic tally of the number of times a given statement has been executed for a student. The author may code a list of branches for an answer-processing instruction, and the appropriate one will be selected for each passage of the student. (When the list runs out, the last branch is used repeatedly for subsequent passages.)

Although these automatic features are potentially very useful for authors, they do not suffice for all needs. The example was deliberately simplified to illustrate the "feel" of CAN-4 and to show that a subset of only four instructions can provide more complex branching than any type of programmed text or so-called "teaching machine" not under computer control. In fact, a slightly expanded subset with automatic scoring facilities is suitable for use by classroom teachers, who can use it with only 1 or $2 \mathrm{~h}$ of instruction. A more rigorous description of the language and some of its features will be given now under the headings: terminal I/O control, response acceptance and recognition, program control and logic, and computation.

\section{LANGUAGE CHARACTERISTICS} Terminal I/O Control

Although acceptance of student responses is an automatic function of answer-list instructions, many more facilities are needed in order to deal with environments more complex than a simple Teletype. CAN-4 provides for output through Teletypes and other keyboard-driven printers, and through CRTs and slide projectors. The OD (output device) instruction initializes an output stream so that all subsequent text messages output by 


\begin{tabular}{ll}
\hline @ & Output student's last response \\
$@ B$ & Ring Teletype bell \\
$@ D$ & Delete current display trom the CRT screen \\
$@ E$ & End the line without carriage return or line spacing \\
$@ I V n$ & Output variable n as integer \\
$@ N$ & Output student's name \\
$@ T$ & Output tab \\
$@ V n$ & Output variable n as decimal number \\
@n & Output n line spaces \\
\hline
\end{tabular}

the $\mathrm{T}$ instruction are directed to the appropriate device. A range of modifiers (Table 2) may be inserted as part of the text string of the $T$ instruction to control formatting or insert string variables in the text (@N-student name; @A-last student response; @Vn-a floating point variable; @IVn-a variable converted to integer format). The PL instruction plots points, vectors, or connected vectors on a CRT screen. Slides are selected (SS-select slide) by a simple preassigned number; a similar system will probably be used for controlling random-access audio output units now under development at The OISE. At present, all student responses are accepted from a keyboard.

For psychological experimentation, where it is frequently important that the $E$ should be able to set certain conditions for input, instructions exist for setting a maximum allowable response time (RT-response time) or a maximum number of key depressions per response (RL-response length). When response matching against answer lists is not required (such as certain cases where the response and the associated latency time are recorded for later analysis), the author may use the read keyboard (RK) instruction which accepts a response from the keyboard and places it without further processing in the student response buffer.

Response Acceptance and Recognition

As mentioned earlier, answer lists consist of one or more anticipated answers followed by a terminator code that is reached when no anticipated response has been found. The $A$ and $U$ operation codes correspond to these functions. However, as a great majority of authors of CAI materials require a simple method of score keeping, three additional answer list operation codes exist; $\mathrm{C}$ for anticipated correct answer, I for anticipated incorrect answer, and W (wrong) for unanticipated answers. A match on one of these codes, or a default match for unanticipated, results in a counter ("variable") being incremented by one (Counter V1 for C, V2 for I, and V3 for W).

In many cases it may serve an author's purpose to accept as correct answers only those which are exactly as specified, without omission of a single character. However, in a far larger number of cases, it is less important to know if a response is typed in $100 \%$ correctly than to recognize the meaning of a response and to categorize it accordingly. Indeed, this is the only means of achieving what may be called "naturalness" in the terminal interaction. All persons who have done computer programming are familiar with the mountain-out-of-a-molehill feeling caused when a missing comma or a bad punch in a control card results in an aborted run. CAN-4 authors also have to live with this picky approach to program writing to some extent, but the language at least allows them the freedom to be much more indulgent with their students' responses. This is achieved by the use of an answer-processing mode switch (Operation Code M), which allows for exact matching (blank modifier or default option), removal of spaces from the response before matching ( $R$ modifier), replacement of punctuation with spaces before matching ( $P$ modifier), searching for keywords or keystrings ( $\mathrm{K}$ modifier), and matching by similarity according to Demerau's (1964) algorithm (S modifier). Subject to the requirement of logical consistency, the modes may be intermixed as required, e.g. keyword/string matching after removal of punctuation. If it is necessary to check for multiple keywords, the author codes the program so that a check is done for one key word and a branch occurs to a second instruction where a scan begins for the second. However, since under normal conditions the contents of the response buffer is deleted on a successful match, a mode exists (I modifier) to inhibit this. If the sequence of the keywords is important, another inhibit mode (C modifier) keeps the character pointer in the student response buffer from being reset before the beginning of the second keyword scan. Instead of multiple keyword searches, in many cases ordered sequences of words like "1492 ... Columbus ... America" can be found in a simpler fashion by inserting a dummy filler (@\#) between significant words in the anticipated response, e.g.,

\section{A,1492@\#COLUMBUS@\#}

AMERICA@\#;nn[branch address]

Although these methods are far from the ideal of true natural language interaction, they are efficient in terms of machine processing time and are a powerful tool in the hands of the skilled program author.

\section{Program Control and Logic}

Branching between sections or lines of a program and between programs is carried out by a variety of specialized instructions. The major unconditional branches are the simple $G$ (go to) statement, the $S$ command to go to a subroutine from which the $R$ (return) command is the normal exit, and the TE (transfer external) command for closing off the program entirely and beginning another. The subroutine (S) instruction can address subroutines in an entirely different program from that in which the call originates; it differs from the transfer external instruction in that the latter does not save a return address. Because of space limitations on the stack of return addresses, nesting of the subroutines is not permitted to a depth of more than 10. In practice, authors seldom utilize nests of more than 2 or 3 subroutines.

Other methods of program control, akin to unconditional branches, are provided by the interrupt address (IA) and restart address (RA) instructions. The IA instruction allows the author to specify a routine to which control transfers whenever the student hits the break key (†P). The return (R) command may subsequently be used to return the student to the interrupted statement. The RA command may be inserted at suitable points in a program where, in case the student signs off before completing the program, the program may later be restarted. The instruction saves all program status indicators.

Conditional branching is accomplished by three means. The first of these, the inclusion of branch lists in the answer list instructions, has already been described. For numerically oriented programs, the CN (convert numeric) instruction allows the author to determine if a digit exists in the answer and, if one exists, to extract the first numeric field from the student response and store it in a specified variable. The generalized conditional branch is the IF instruction which tests the relationship (greater than, less than, equals, etc.) between two variables (counters). If the relationship is true, a branch 
occurs. The branch address is selected from a branch list of the same type as that used for the answer list instructions. The instruction makes it possible to control program flow on the basis of relationships varying from the very simple to the very complex. The possibility for complex logic depends in large measure on the use of the IF instruction to test results obtained by means of the $\mathrm{CO}$ (compute) operation.

\section{Computation}

The computation facility is designed for several types of uses: generating and scoring arithmetic problems; graph plotting on the CRT; complex decision making regarding the presentation of instructional materials; and permitting student access to a "desk calculator" facility. CAN-4 makes available a total of 51 separate numerical counters (V0.V50). Four counters are used for system functions: one (V0) for accumulating total user "sign on" time and three for automatic scoring operations (C, correct: V1; I, incorrect: V2; W, unanticipated wrong: V3). Except for V0, which is write-protected, the counters may be used as temporary storage in computation or for storage of results. Computation is carried out in double-precision floating point under control of statements similar to FORTRAN or BASIC. The FORTRAN operators for addition (+), subtraction (-), multiplication $(*)$, division (/), and exponentiation $(* *)$ are provided. If required, the author may call a random value or the current value (in seconds) of the time-of-day clock and may convert values from floating point to integer format by truncation. In order to save time and space, a few limitations have been introduced: Computation proceeds from left to right without hierarchy of operations, except as provided by the use of parentheses; the nesting of parentheses is not permitted. The instruction format has also been modified to conform to that found in other CAN-4 statements: the mnemonic $\mathrm{CO}$ is separated by a comma from the name of the variable where the result is to be stored; this, in turn, is separated by a second comma from the body of the statement describing the computation. Curriculum authors activate the desk calculator facility by using a dummy operator which causes a student response to be evaluated as if it were the expression field of the compute statement. Despite its limitations, the facility is quite flexible and has sufficed for all needs encountered thus far in our course development work.

\section{CONCLUSION}

The most important evaluation criterion for an author language is its utility to authors in aiding them to design more effective and flexible instructional materials. Our experience with CAN-4 has so far been limited to use by staff and students at The OISE. But even this limited trial has demonstrated conclusively that the language provides significantly more power than earlier versions in the CAN language series. However, this power has not been bought at the price of making the language inaccessible to the curriculum authors it is intended to serve. It is still possible in 2 or $3 \mathrm{~h}$ of instruction to teach novices all the language syntax required for complex programming. By retaining the basic characteristics of our earlier languages in CAN-4, it has been possible to assure compatibility of programs. Materials programmed in the earlier languages of the CAN series can be reformatted automatically by utility programs to run as CAN-4 programs. During the coming year this language facility will be tested under operational conditions as part of in-school CAI projects.

\section{REFERENCES}

DEMERAU, F. A technique for computer detection and correction of spelling errors. Communications of the ACM, 1964, 7, 3(March), 171-176.

ENSOR, D. A. A time-sharing system for the PDP-9. In: DECUS proceedings, Fall 1969. Maynard, Mass: Digital Equipment Computer Users' Society, 1969.

McLEAN, R. S. TSS-9 revisited. Paper presented at Spring 1970 meeting of Digital Equipment Computer Users' Society, Atlantic City, May 1970. 